\title{
APPLICATIONS FOR ENERGY RECOVERING FREE ELECTRON LASERS
}

\author{
George R. Neil \\ Thomas Jefferson National Accelerator Facility, Newport News, VA 23606 USA
}

\begin{abstract}
The availability of high-power, high-brilliance sources of tunable photons from energy-recovered Free Electron Lasers is opening up whole new fields of application of accelerators in industry. This will review some of the ideas that are already being put into production, and some of the newer ideas that are still under development.
\end{abstract}

\section{MOTIVATION AND BACKGROUND}

Jefferson Lab initiated its Free Electron Laser Program in 1995 with the idea that at suitable power levels a Free Electron Laser would have commercial applications. Such deployment of a laser technology could only take place provided R\&D demonstrates there is a commercial market, the process can manufacture sufficient product to supply a viable fraction of the demand, the technical approach works, and the cost of the process and the capital investment is small compared to the payback. We had identified at the onset a range of applications which had potential for commercial viability. The viability of various processes depended on the cost per kilojoule of light delivered including full cost recovery. Based on scaling studies we found that such a cost fell dramatically with the FEL output power [1]. At the $10 \mathrm{~kW}$ level FEL photons were projected to cost on the order of $\$ 0.01 / \mathrm{kJ}$. Since FEL hardware does not scale linearly with output power we also found that by the time the FEL reached $100 \mathrm{~kW}$ the cost would fall to on the order of $\$ 0.002 / \mathrm{kJ}$ opening up many more opportunities for applications.

This, of course, presumes that such a high power FEL could be built and would be practical for use in a manufacturing environment. At the time of the initial studies no FEL had produced more than 10 Watts of average power placing in doubt the projected cost values as well as the viability of the laser to say nothing of the various possible industrial processes considered. Twelve years have passed and substantial progress has been made in terms of demonstrating FEL capability, validating the cost scaling models, and identifying possible commercial applications. Nonetheless, at this point there is still a need to carry forward the early stage work making use of prototype manufacturing equipment or laboratory scale hardware. The early-stage R\&D must yield enough scientific understanding and practical experience to validate the design of such prototype manufacturing facilities. Demonstration products are also important so that initial estimates of business values and manufacturing costs are possible.
We will spend the rest of this paper reviewing the technical status of high power FELs and discussing some of the specific applications that presently appear promising.

\section{TECHNICAL STATUS}

The JLab IR Upgrade holds the power record for FELs having produced $14.3 \mathrm{~kW}$ of $\mathrm{CW}$ average power at 1.6 microns. It has also produced substantial powers at wavelengths as long as 10 microns and as short as 1 micron [2]. The details of that system and its performance are beyond the scope of this paper but we will briefly describe the system to highlight the components critical to high average power production.

The system is illustrated in Figure 1. The Upgrade is comprised of a DC gun and superconducting rf cavity injector producing up to $10 \mathrm{MeV}$ of acceleration for up to $10 \mathrm{~mA}$ of average current in $74.85 \mathrm{MHz}, 135 \mathrm{pC}$ pulses in a continuous pulse train. Following the injector three superconducting 8-cavity modules accelerate the beam to $120 \mathrm{MeV}$ whereupon it is injected into a permanent magnet wiggler with 30 periods of $55 \mathrm{~mm}$. At the wiggler entrance the electron beam pulses are less than $400 \mathrm{fs}$ FWHM. About $1 \%$ of the electron beam power is extracted as light into a 32 meter long optical cavity, outcoupled through a partially transmissive mirror, and brought to one of a set of 7 User Labs for application. The remaining $\sim 99 \%$ of the beam power is recycled back to the accelerator by returning the electron bunches approximately 180 degrees out of phase. The beam is decelerated back down to its injection energy and sent to a water cooled beam dump [3]. The beam power is thus supplied back to the rf field reducing the required drive power by a large factor. In addition, significant technical benefits accrue from the lower power on the beam dump. Even more beneficial is the elimination of neutron production and thereby component activation because the dump beam energy is below the photoneutron threshold. This Energy Recovering Linac (ERL) approach has been so successful that a number of laboratories around the world are developing programs for $100 \mathrm{~mA}$, high energy $(>500 \mathrm{MeV}$ ) versions as photon sources for fundamental and applied scientific research $[4,5]$.

Substantial progress has been made using this device to quantify the operational limits and optimize the design. Ongoing Navy interest in producing high average power FELs is providing substantial industrial program synergy. 
The Navy requirements include $100 \mathrm{~kW} \mathrm{CW}$ average power at wavelengths around 1 micron, a compact, reliable and robust design, cost effectiveness, and ease of operation [6]. We have a conceptual design for such a
$100 \mathrm{~kW}$ system as illustrated in Figure 2 [7]. Note that progress in ERL and srf technology permits a factor of 10 scaleup in power in a system $1 / 3$ the size utilizing only a single superconducting linac module. The average
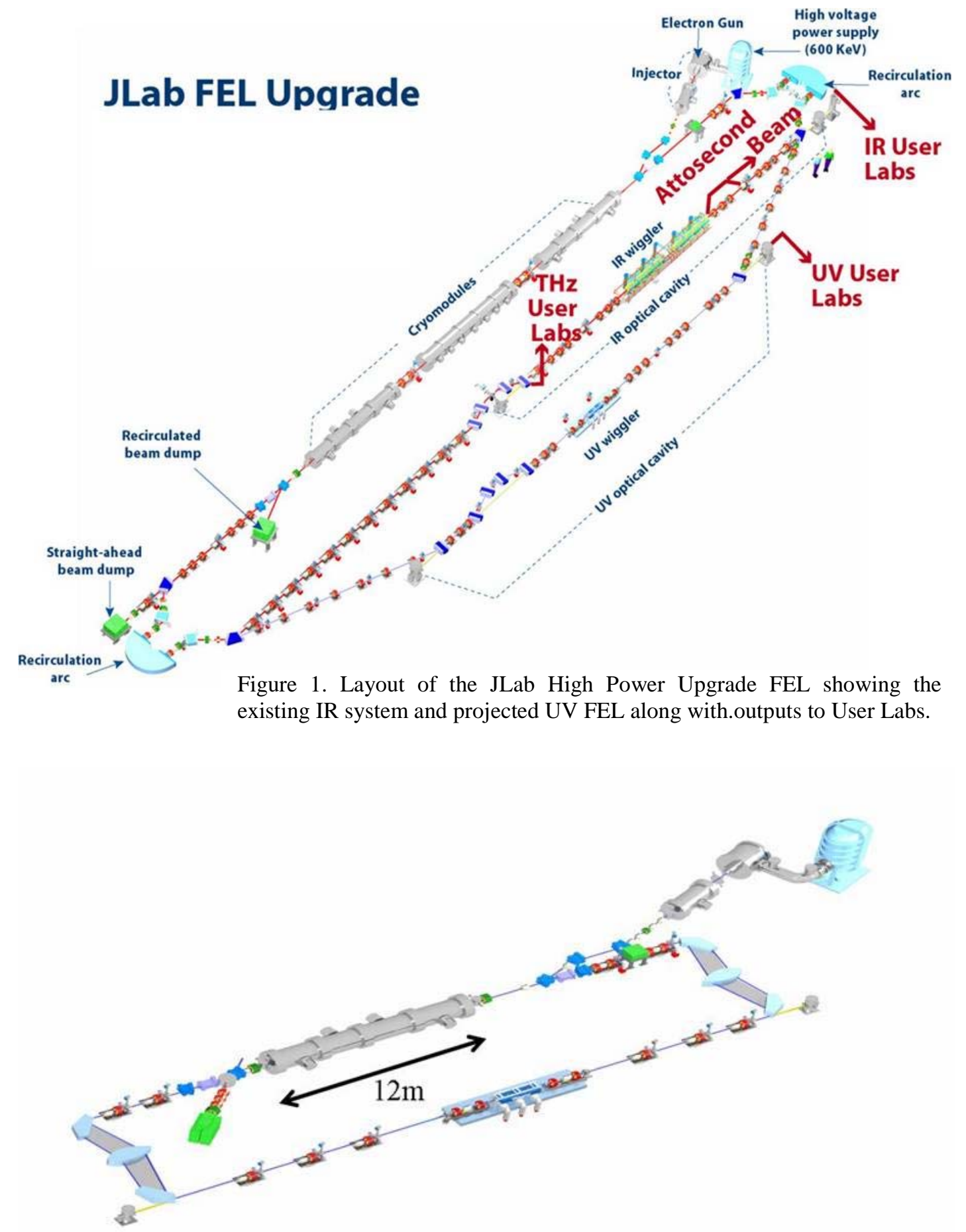

Figure 2. A compact FEL incorporating advances in the design of srf modules and better understanding of electron beam transport in energy recovering systems. 
current in such a system would be about $70 \mathrm{~mA}$ and the output voltage around $100 \mathrm{MeV}$. If the linac frequency were kept the same as in the present system $(1497 \mathrm{MHz})$ then the higher average power could be produced simply by increasing the pulse repetition rate from $74.85 \mathrm{MHz}$ to $748.5 \mathrm{MHz}$ and keeping the charge/pulse constant. This means the FEL extraction efficiency should also remain the same and the 10 times higher current yields 10 times the output power. Since most physics issues dealing with beam quality scale with the charge and not the average current this lends some credence to the design specification. There are, of course, average current issues which must be dealt with: injector rf power handling, beam breakup, resistive wall heating, and HOM power damping [8]. This remains a field of active study around the world and progress is expected over the next few years on many fronts. Perhaps the most challenging issue to get to $100 \mathrm{~kW}$ output powers is creating the initial high average brightness beam at the injector. It is also worth noting that the short pulses of high charge can produce copious amounts of short pulse, broadband $\mathrm{THz}$ radiation [9]. Potential applications of this radiation are a fertile field of research but it is beyond the scope of this paper to discuss imaging, remote sensing, etc.

One aspect of system level progress is particularly encouraging: cost estimates have dropped considerably since the initial scaling was presented. In the original study a second item (ignoring non-recurring engineering) $100 \mathrm{~kW}$ FEL was estimated at \$35M in 1995 dollars. We now believe the second item $100 \mathrm{~kW}$ FEL can be built for around $\$ 30 \mathrm{M}$ in 2007 dollars [Figure 3]. The reason for this cost reduction is the substantially higher gradients that can be achieved from srf cavities thereby reducing the number of cavities and rf systems required. It is also now technically feasible to inject at lower energy saving both capital investment in $\mathrm{rf}$ power and reduced electricity costs during operation. Thus we have continued interest in developing the FEL for applications [10-13].

\section{POTENTIAL APPLICATION}

The JLab IR Upgrade FEL has enabled a rich applied and basic science program that included the investigation of both linear and nonlinear phenomena in proteins, ceramics, and metals, pulsed laser ablation and deposition, laser nitriding, synthesizing carbon nanotubes, and micromachining. Linear dynamics using pump-probe techniques was applied to amide-I absorption at 5 to $8 \mu \mathrm{m}$ in proteins [14] and to hydrogen defects in silicon at $3 \mu \mathrm{m}$ $[15,16]$. Medical research at the FEL resulted in development of an approach to treating adult acne through the selective necrosis of sebaceous fat glands without harm to the surrounding skin [17]. In the following we expand on some of these applications of a high power, high repetition rate IR FEL and by extension suggest areas that may be profitable for industrial application.

Pulsed laser ablation and deposition (PLD) is a potential application for manufacturing large-area films. The FEL's ultrafast pulses offer a low ablation threshold, substantially lessened target damage, and particulate elimination. High repetition rate implies high deposition rate, and may mean greater control over ablation and growth dynamics. Wavelength tunability results in enhanced ablation and deposition with resonant absorption in molecular materials such as in polymers. We have demonstrated PLD of metals such as niobium and steel [18] and shown that the qualities of the deposited material are superior to standard laser deposition.

There has also been substantial work on IR PLD of polymers at Vanderbilt and our FEL [21]. Using resonant IR excitation of selected vibrational modes, the IR photons transfer their energy to specific molecular degrees of freedom. These anharmonic vibrations couple relatively slowly to the harmonic vibrations that constitute the phonon bath. Typical relaxation times for such anharmonic excitations are in the 1-10 ps range, so that nuclear motion and bond-breaking can begin, if the density of excitation is sufficiently high, before the energy

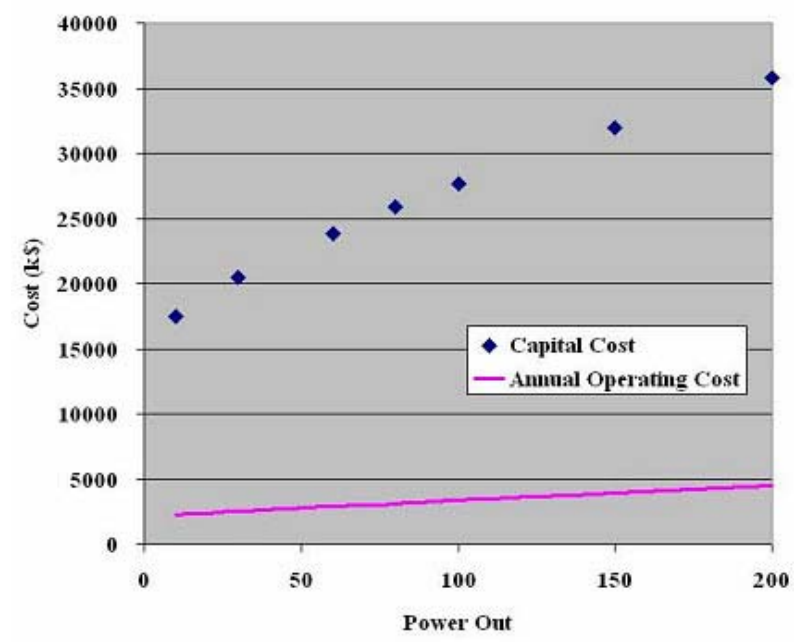

Figure 3. Capital and operating cost for an FEL as a function of FEL power. The operating cost includes

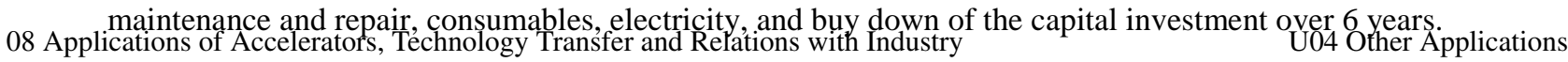


leaks out of the excited mode. Moreover, vibrational excitation also does not generate electronic excitations, and thus avoids relaxation pathways that lead to photofragmentation or structural alterations, as confirmed by the XPS measurements. A superior polymer coating results. A new pulsed laser deposition facility is in the process of being installed in the FEL User lab to exploit advances in our understanding of these processes.

Laser nitriding is a method of modifying the properties of metals to obtain a harder surface with better corrosion resistance and the ability to hold higher standoff voltages. The JLab FEL was successfully used to produce high quality nitride films on iron, titanium, and silicon [20, 21]. Most interestingly the work showed successful nitriding could be achieved at atmospheric pressure without resort to a vacuum chamber. This implies much lower process costs in an industrial setting. The character of the surface produced also showed improved characteristics over that produced with excimer lasers.

The sub-picosecond pulse structure of the FEL can be used to good advantage to melt the surface layer of a metal such as ordinary steel. Because the pulse is so short cooling occurs very rapidly, $10^{15} \mathrm{~K} / \mathrm{sec}$. As a consequence the metal surface becomes amorphous and exhibits enhanced tensile strength and corrosion resistance. Example applications would include turbine blades, automotive components, or structures exposed to sea environments.

Carbon nanotube structures present a range of production challenges that might be circumvented or better understood by use of the JLab FEL's repetition rate, wavelength tuning and power. Studies were performed to determine how the structures are formed to discover optimum conditions for production with tailored properties, as well as to learn more about real-time process monitoring and control. The JLab FEL with $3 \mu \mathrm{m}$ light at 400 to $600 \mathrm{~W}$ average power synthesized single-wall carbon nanotubes with smaller diameters than nanotubes produced by direct current arc or table-top pulsed laser vaporization. Initial production rates were measured in milligrams per minute rather than milligrams per hour [22]. Through studies of the production mechanisms we have now extended that initial work and shown production of many grams per hour of high quality, highly pure, single wall nanotubes and are investigating extensions into other sorts of materials such as boron. This production is already cost effective (value of product substantially exceeds recovery cost of operating the FEL) for an industrial process and will improve further as the technique is optimized and FEL powers increase.

Micromachining is applied to metals for automotive engine applications, and on glasses and ceramics for the fabrication of truly three-dimensional microstructures. With nano-scale engineering of key features, it is hoped that satellites could be reduced to the size of baseballs or smaller [23]. We intend to explore the development of this application more extensively using the output of the UV Upgrade presently under construction. Production rates should be significantly enhanced over available excimer laser systems and the range of materials that can be employed will be greatly expanded. We have installed and brought into initial operation a laser microengineering tool to permit CAD control of the laser beam to directly micromachine or even micro-engineer objects up to $30 \mathrm{~cm}$ x $30 \mathrm{~cm}$ x $50 \mathrm{~cm}$ with a resolution better than 10 microns (see Fig. 4). This facility can operate in both the IR and UV and can be controlled remotely over the internet. The short pulse character of the FEL should offer substantially enhanced ability to micromachine ceramics or refractory materials.

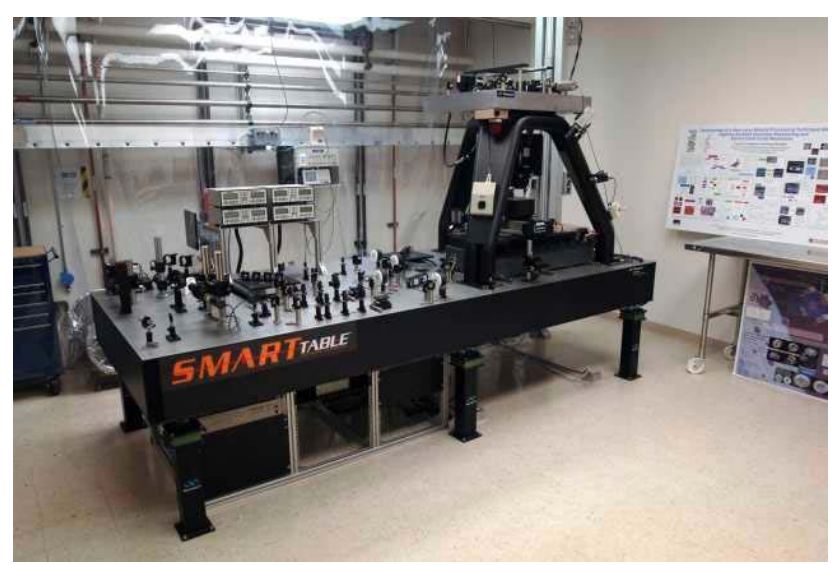

Figure 4. The JLab Laser Microengineering Station. This system provides state of the art positioning of an intensity controlled laser beam as well as write velocity control to ensure equal exposure. The system is programmable from converted CAD drawing files. Although we do not yet have the UV FEL operational we have demonstrated high power upconversion to the UV of 1 micron lasing which can be used as a source at the required powers.

\section{SUMMARY}

To have prospect of entry into manufacturing, the FEL must deliver good economics as well as attractive performance. The economics scale favourably with high power output. The extension of FELs to higher powers will benefit from ongoing research in ERL technology for scientific applications. A number of groups are pushing toward the $100 \mathrm{~mA}$ average current high brightness injector capable of driving a $\sim 100 \mathrm{~kW}$ FEL. Using that injector with present srf technology a single cryomodule in a ERL configuration could provide the beam energy required for IR output. This could form the basis of a highly compact and robust FEL supporting any number of possible applications as described above. In addition the initial cost analysis promises to deliver FEL output at the cost of $\$ 0.05 / \mathrm{kJ}$ to $\$ 0.002 / \mathrm{kJ}$, a number competitive with many other specialized industrial lasers, so that as this technology matures we expect to see industrial entry into the FEL field to join the already vibrant scientific activity. 


\section{ACKNOWLEDGEMENTS}

Jefferson Lab is supported by the U.S. Dept. of Energy under contract DE-AC05-84-ER40150. The FEL is supported by the Office of Naval Research, the Air Force Research Laboratory, the Commonwealth of Virginia and the Laser Processing Consortium. The work cited in this paper is the result of efforts by the FEL Team without whose work I would have little to say. I am grateful for their continued help. I would especially like to thank Michael J. Kelley for his help in describing the cited applications in this manuscript.

\section{REFERENCES}

[1] G. R. Neil, "A Cost Estimation Model for High Power FELs,” Proc. 1995 Particle Accelerator Conference and Conference on High Energy Accelerators (1996) IEEE 137.

[2] G. R. Neil, C. Behre, S. V. Benson, M. Bevins, G. Biallas, J. Boyce, J. Coleman, L.A. Dillon-Townes, D. Douglas, H. F. Dylla, R. Evans, A. Grippo, D. Gruber, J. Gubeli, D. Hardy, C. Hernandez-Garcia, K. Jordan, M. J. Kelley, L. Merminga, J. Mammosser, W. Moore, N. Nishimori, E. Pozdeyev, J. Preble, R. Rimmer, M. Shinn, T. Siggins, C. Tennant, R. Walker, G. P. Williams, and S. Zhang, "The JLab High Power ERL Light Source," Proceedings of 32nd Advanced ICFA Beam Dynamics Workshop on Energy Recovering Linacs, Jefferson Lab, March 19-23, 2005.

[3] G. R. Neil, S. Benson, G. Biallas, C. L. Bohn, D. Douglas, H. F. Dylla, R. Evans, J. Fugitt, A. Grippo, J. Gubeli, R. Hill, K. Jordan, R. Li, L. Merminga, P. Piot, J. Preble, M. Shinn, T. Siggins, R. Walker, B. Yunn, "Sustained Kilowatt Lasing in a Free-Electron Laser with Same-Cell Energy Recovery," Phys. Rev. Lett. 84 (2000) 662.

[4] S. L. Smith, D. J. Holder, P. McIntosh, N. Bliss, "The Status of the Daresbury Energy Recovery," PAC'07, New Mexico, June 2007, Paper TUPMN084.

[5] G. Hoffstaetter, I. V. Bazarov, G. Codner, M. Forster, S. Greenwald, Y. Li, M. Liepe, C. Mayes, C. K. Sinclair, C. Song, A. Temnykh, M. Tigner, Y. Xie, D. H. Bilderback, D. Dale, K. Finkelstein, S. M. Gruner, "Progress Toward an ERL Extension to CESR," PAC'07, New Mexico, June 2007, Paper MOOBAB02.

[6] J. Blau, T. Campbell, W. B. Colson, W. Ossenfort, G. R. Neil, S. V. Benson, H. F. Dylla and M. D. Shinn. "Simulations of the $100 \mathrm{~kW}$ TJNAF FEL using a short Rayleigh Length," Nucl. Instr. and Meth. in Phys. Res. A483 (2002) 142-145.

[7] D. Douglas, K. Beard, S. V. Benson, P. Evtushenko, C. H.-G., S. W. Moore, G. R. Neil, T. Powers, C. Tennant, "A Compact ERL Driver for a High-Power
IR FEL,’ PAC'07, New Mexico, June 2007, Paper TUPMS061.

[8] G. R. Neil and L. Merminga, "Technical Approaches for High Average Power FEL," Reviews of Modern Physics 74, (2002) 685.

[9] G. P. Williams, "FarIR/THz Radiation from the Jefferson Lab FEL Energy Recovered Linac," Rev. Sci. Instr. 73 (2002) 1461.

[10]H. F. Dylla, Laser Focus World, August 2001.

[11] M. J. Kelley, et al., SPIE Proc. Issue 2703 (1996) 1520.

[12] G. R. Neil, "Industrial Applications of the Jefferson Lab High-power Free-electron Laser," Nucl. Instr. and Meth. in Phys. Res. B 144 (1998) 40-49.

[13] H. F. Dylla et al., SPIE Vol. 3925 (2000) 40-49.

[14] R. H. Austin, A. H. Xie, L. van der Meer, M. Shinn, G. Neil, "Self-trapped States in Proteins" Nucl. Instr. and Meth. in Phys Res. A507 (2003) 561.

[15] M. Budde, et al., Phys. Rev. Lett. 85, (2000) 1452.

[16] G. Luepke, X. Zhang, B. Sun, A. Fraser, N. H. Tolk and L. C. Feldman "Structure-Dependent Vibrational Lifetimes in Silicon," Phys. Rev. Lett. 88 (2002) 135501.

[17]R. R. Anderson, W. Farinelli, H. Laubach, D. Manstein, A. N. Yaroslavsky, J. Gubeli III, K. Jordan, G. R. Neil, M. Shinn, W. Chandler, G. P. Williams, S. V. Benson, D. R. Douglas, H. F. Dylla, "Selective Photothermolysis of Lipid-rich Tissues: A Free Electron Laser Study," Lasers in Surgery and Medicine, Volume 38, Issue 10, (2006) 913-919.

[18] A. Reilly, C. Allmond, S. Watson, J. Gammon, J. G. Kim, "Pulsed Laser Deposition with a High Average Power FEL," J. Appl. Phys. 93 (2003) 3098.

[19]D. M. Bubb, J. S. Horwitz, R. A. McGill, D. B. Chrissey, M. R. Papantonakis, R. F. Haglund, B. Toftmann; "Resonant IR PLD of a Sorbent ChemoSelective Polymer," Appl. Phys. Let. 79 (2001) 2847.

[20] E. Carpene, P. Schaaf, M. Han, K. P. Lieb and M. D. Shinn. "Reactive Surface Processing by Irradiation with Excimer Laser, Nd:YAG Laser, Free Electron Laser and Ti:sapphire Laser in Nitrogen Atmosphere," Appl. Surf. Sci. 186 (2002) 195-199.

[21]E. Carpene, M. D. Shinn and P. Schaaf, "Synthesis of Highly Oriented TiN Coatings by Free Electron Laser Processing of Titanium in Nitrogen," Appl. Phys. A 80 (2005) 1707-1710.

[22] P. C. Ecklund, B. K. Pradhan, U. J. Kim, Q. Xiong, J. E. Fischer, A. D. Friedman, B. C. Holloway, K. R. Jordan and M. W. Smith. "Large-scale production of single-walled carbon nanotubes using ultrafast pulses from a free electron laser," Nano Letters 2 (2002) $561-566$.

[23] H. Helvajian (Ed.), Microengineering Aerospace Systems, AIAA, Reston, VA (1999). 